\title{
Effects of Forming Processes on Microstructures and Mechanical Properties of A356 Aluminum Alloy Prepared by Self-inoculation Method
}

\author{
Li Ming ${ }^{a}{ }^{\circledR}$, Li Yuan-dong ${ }^{b}$, Yang Wen-long ${ }^{a}$, Zhang Yu ${ }^{a}$, Wang Zong-gang ${ }^{a}$ \\ ${ }^{a}$ Hexi University, Zhangye 734000, China \\ ${ }^{b}$ State Key Laboratory of Advanced Processing and Recycling of Nonferrous Metals, Lanzhou University \\ of Technology, Lanzhou 730050, China
}

Received: October 30, 2018; Revised: December 07, 2018; Accepted: March 09, 2019

\begin{abstract}
Semisolid slurry of A356 aluminum alloy was prepared by Self-Inoculation Method (SIM), and the microstructures and solidification behavior of rheo-forming process in different forming processes were researched. The results indicate that semisolid slurry of A356 aluminum alloy can be prepared by Self-Inoculation Method at $600^{\circ} \mathrm{C}$. Primary $\alpha$-Al particles with fine and spherical morphology are uniformly distributed when the isothermal holding time of slurry is $3 \mathrm{~min}$. The different forming processes for semisolid rheo-forming can be regarded as the effect of cooling rate on rheo-forming. The primary particle sizes and shape factors are gradually increasing with the increase of cooling rate. The sizes of secondary particles are gradually increasing with the decrease of cooling rate, and the amount of secondary particles in the same areas are gradually decreasing with the decrease of cooling rate. The morphologies of eutectic Si are gradually coarsening from fibrous to needle-like in different forming processes, while the sizes and the lamellar spacing of the eutectic Si are gradually increasing with the decrease of cooling rate. The mechanical properties of the semisolid forming components are gradually increasing with the increase of cooling rate and forming pressure.
\end{abstract}

Keywords: A356 aluminum alloy, semisolid, self-inoculation method, solidification behavior, eutectic structure.

\section{Introduction}

Semisolid metal processing has been widely recognized by experts and scholars since it is first developed, and is regarded as the most promising modern processing technology in the 21 st Century ${ }^{1,2}$. After more than 40 years of exploration and research, scholars successively developed preparation process of semisolid microstructure, such as the twin-screw slurry maker ${ }^{3}$, GISS (The Gas Induced Semisolid) ${ }^{4}$, SCP (serpentine pouring channel) ${ }^{5}$, WSP (wavelike sloping plate) ${ }^{6}$, SSR (Semi-solid Rheocasting) ${ }^{7}$, CRP (Continuous Rheoconversion Process) ${ }^{8}$, NRC (New Rheocasting Process) ${ }^{9}$, SEED (Swirled Enthalpy Equilibration Device) ${ }^{10}$ and so on. Based on the characteristics of low superheat pouring, liquid-liquid mixed casting, solid-liquid mixed casting, suspension casting and inclined cooling method, a new type of solidification structure control method is proposed, which called Self-inoculation Method (SIM) ${ }^{11}$.

Semisolid metal rheological forming is a direct forming method using solid-liquid mixed slurry with two solidification process. The process of slurry preparation (solid particles precipitate from liquid alloy) is called primary solidification, and the solidification process of slurry in the subsequent forming (solidification of remaining liquid) is called secondary solidification. In recent years, the study of semisolid metal rheological forming mainly focused on primary solidification,

*e-mail: 996751102@qq.com while the research of secondary solidification was not too much $^{12-16}$. While the studies have indicated that the solidification of remaining liquid in semisolid slurry has an important influence on the final solidification microstructures and mechanical properties of alloy. Therefore, it is necessary to study the precipitation behavior of the secondary particles in the remaining liquid of the slurry, the eutectic reaction and their microstructural characteristics. In view of this, based on the previous researches ${ }^{17-20}$, the rheo-forming of A356 aluminum alloy was prepared combining semisolid slurry preparation by self inoculation method with different forming process. Microstructures of both primary solidification and secondary solidification in different forming processes were analysed. The relationship between different cooling rate and the solidification microstructures was explored. Solidification behavior of remaining liquid in different forming processes of A356 aluminum alloy was elaborated by quantitative analysis of secondary particles and the Si morphology in different forming components, in order to provide theoretical reference and technical guidance for rheo-forming application of A356 aluminum alloy.

\section{Experimental detail}

\subsection{Preparation of self-inoculants}

A commercial unmodified A356 aluminum alloy (actual composition is shown in Table 1) was melted in a pit-type 
Table 1. Chemical composition of commercial A356 alloy (wt\%)

\begin{tabular}{ccccccc}
\hline $\mathrm{Si}$ & $\mathrm{Mg}$ & $\mathrm{Fe}$ & $\mathrm{Ti}$ & $\mathrm{Cu}$ & $\mathrm{Zn}$ & $\mathrm{Al}$ \\
\hline 7.06 & 0.27 & 0.115 & 0.097 & 0.001 & 0.01 & Balance \\
\hline
\end{tabular}

electric resistance furnace. The melt was degassed by $\mathrm{C}_{2} \mathrm{Cl}_{6}(1$ mass $\%$ of alloy) at $720^{\circ} \mathrm{C}$, then cooled to $700^{\circ} \mathrm{C}$ and poured into a metal mold to obtain the metal bars with the size of $\Phi 15 \mathrm{~mm} \times 150 \mathrm{~mm}$. Then the bars were machined into small particles with sizes of about $5 \mathrm{~mm} \times 5 \mathrm{~mm} \times 5 \mathrm{~mm}$.

\subsection{Slurry preparation and optimal parameter selection}

Fig. 1 shows the schematic diagram of slurry preparation by SIM (self-inoculation method). The fluid director was inclined at $45^{\circ}$ with a length of $500 \mathrm{~mm}$. The commercial A356 alloy was melted and degassed, then adjusted the melt temperature to $680^{\circ} \mathrm{C}$, added $5 \%$ (mass fraction of the melt) inoculants into the melt and stirred with iron bars quickly to make them dissolved. Then the mixed melt was collected through fluid director to the slurry collector to obtain semisolid slurry, and directly poured into cold water to obtain waterquenched specimens without isothermal holding. Finally, the prepared slurry was isothermally held for a certain time (0 $\mathrm{min}, 3 \mathrm{~min}, 5 \mathrm{~min}$ and $10 \mathrm{~min}$, respectively) at $600^{\circ} \mathrm{C}$, then directly poured into cold water to obtain water-quenched specimens with different isothermal holding parameters. The optimal parameters were selected by observing the optical microstructures of the semisolid slurry (average particle sizes and shape factors of primary particles).

\subsection{Rheo-forming, microstructure observation and quantitative analysis}

The rheo-forming were carried out after selecting the optimal parameters of the semisolid slurry. The selected semisolid slurry was poured into different mould with different cooling rate to form different parts. Then the parts were processed to small cubic aluminum specimens. The specimens were prepared by the standard technique of grinding with $\mathrm{SiC}$ abrasive paper and polishing with an $\mathrm{Al}_{3} \mathrm{O}_{2}$ suspension solution, followed by etching in saturated $\mathrm{NaOH}$ aqueous solution. Then the MFE-4 Optical microscopy (OM) was used to observe the microstructures. The average particle sizes $(D=(4 A / \pi) 1 / 2$, where $A$ is area of the particle) and shape factors $(F=P 2 /(4 \pi A)$, where $P$ is the perimeter of particle) of primary particles were measured using image analysis software Image-Pro Plus 5.0. The FEG450 scanning electron microscopy (SEM) was carried out, equipped with an energy dispersive spectroscopy (EDS) facility and operated at an accelerating voltage of $3-20 \mathrm{kV}$ to observe the morphologies of secondary particles and eutectic structures.

Fig. 2 shows the schematic diagram of the temperature change in the experiment process. The inoculants are added when the melt temperature reaches $T_{1}$ and reduced to $T_{2}$. And

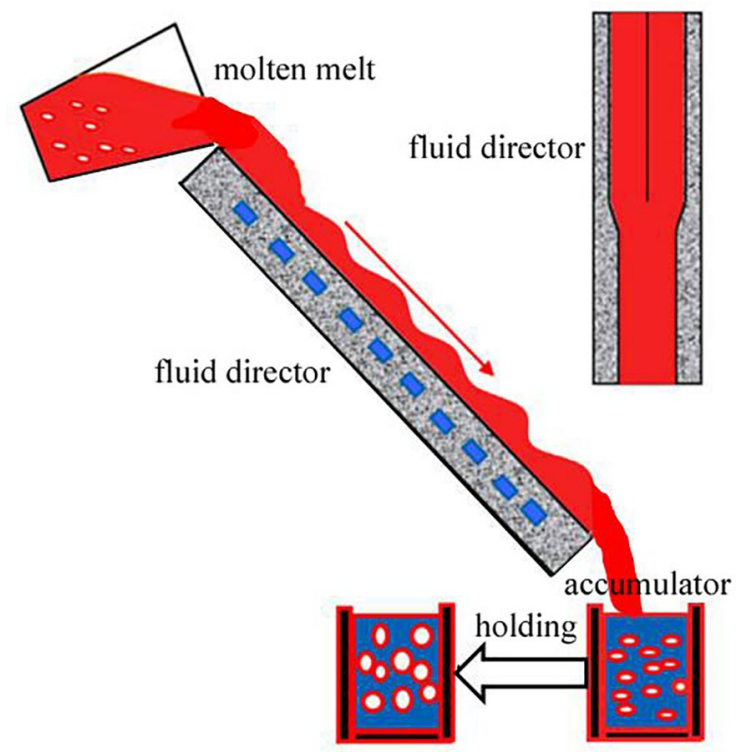

Figure 1. Schematic diagram of slurry preparation by SIM

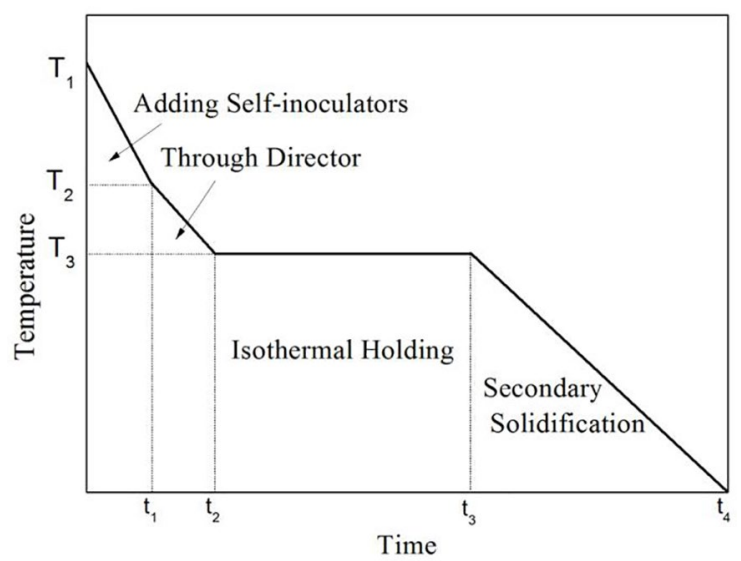

Figure 2. Schematic diagram of the temperature change in semisolid process by SIM

then the temperature is reduced to $\mathrm{T}_{3}$ after the melt flows through the director, and there is a isothermal holding process for a specific time at $\mathrm{T}_{3}$ temperature. Finally, the forming process is carried out. In this experiment, the average cooling $\left(\mathrm{T}_{2}-\mathrm{T}_{1}\right)$ after adding inoculants is about $50^{\circ} \mathrm{C}$, and the temperature drops about $30^{\circ} \mathrm{C}\left(\mathrm{T}_{3}-\mathrm{T}_{2}\right)$ after the melt flowing through the director. Thus, the mean temperature change in the whole process of SIM is reduced by about $80^{\circ} \mathrm{C}$. In the process of SIM due to the influence of latent heat of crystallization, the higher the melt treatment temperature, the higher the temperature changes. The mean temperature change in this case is to be used as a reference value for presetting holding temperature of different melt treatment, such as the melt treatment temperature of $690^{\circ} \mathrm{C}$ corresponding to the holding temperature of $610^{\circ} \mathrm{C}$. Therefore, the setting value of the heat preservation temperature in this experiment is $80^{\circ} \mathrm{C}$ below the melt treatment temperature. 
Fig. 3 shows the sizes of the samples in different forming processes. It can be seen that the measured cooling rates of the different samples are $1000 \mathrm{~K} / \mathrm{s}, 17.2 \mathrm{~K} / \mathrm{s}, 5.2 \mathrm{~K} / \mathrm{s}$ and $2.9 \mathrm{~K} / \mathrm{s}$, corresponding to rheo-diecasting with thickness of $2 \mathrm{~mm}$, permanent mould casting with diameter of $15 \mathrm{~mm}$, permanent mould casting with diameter of $50 \mathrm{~mm}$ and sand mould casting with diameter of $50 \mathrm{~mm}$, respectively.

\section{Results and Discussions}

\subsection{Parameters optimization of semisolid slurry preparation}

The water-quenched microstructures of A356 aluminum alloy semisolid slurry after isothermal holding at $680^{\circ} \mathrm{C}$ for $0 \mathrm{~min}, 3 \mathrm{~min}, 5 \mathrm{~min}$ and $10 \mathrm{~min}$, respectively are shown in Fig. 4. It shows that the semisolid slurry containing roseshape and fine dendritic primary particles can be prepared by SIM (as shown in Fig. 4(a)). After isothermal holding for a short time (such as $3 \mathrm{~min}$ and $5 \mathrm{~min}$ ), the dendrite arms of primary particles are fused, and primary particles become spherical (as shown in Fig. 4(b)). But when the holding time of slurry is too long (such as $10 \mathrm{~min}$ ), the sizes of primary particles gradually increasing while the merging phenomenon among primary particles occurs obviously, which leads to the emergency of " 8 " shaped and "spindle-like" structures (as shown in Fig. 4(d)).

The effects of different isothermal holding time on average particle sizes and shape factors of primary particles shown in Fig. 5 are measured according to Fig. 4. It illustrates that the primary particle size is minimum with the average value of $36 \mu \mathrm{m}$ when the slurry is not undergoing the isothermal holding process, but the shape factor is the largest with the average value of 1.9. When the isothermal holding time is $3 \mathrm{~min}$, the particle size is increased to $66 \mu \mathrm{m}$ and the shape factor is reduced to about 1.4. When the holding time is increased to $5 \mathrm{~min}$, the average size of the primary particle is increased to $84 \mu \mathrm{m}$. When the holding time is increased to $10 \mathrm{~min}$, the average size of the primary particles is increased

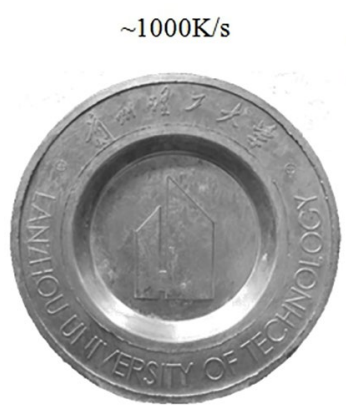

(a)

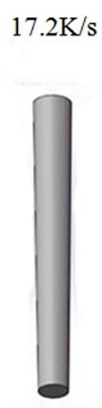

(b)

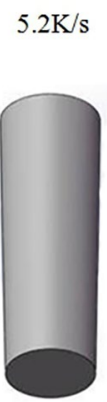

(c)
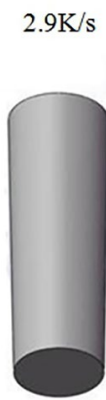

(d)
Figure 3. Schematic diagram of different forming mould (a) rheodiecasting with thickness of $2 \mathrm{~mm}$; (b) permanent mould casting with diameter of $15 \mathrm{~mm}$; (c) permanent mould casting with diameter of $50 \mathrm{~mm}$; (d) sand mould casting with diameter of $50 \mathrm{~mm}$. to $106 \mu \mathrm{m}$, and the shape factor is larger than $5 \mathrm{~min}$ (with the average value of about 1.63).

Fig. 6 shows the linear regression of the growth of the primary particles with different isothermal holding time. It is evident combining with Fig. 5 that primary particles are gradually growing and spheroidizing in the early stage of isothermal holding process, and the growth rate of the primary particles in the isothermal holding process conforms to the dynamic equation of $\mathrm{D}_{\mathrm{t}}^{3}-\mathrm{D}_{0}^{3}=\mathrm{Kt}^{21}$ (where $\mathrm{D}_{0}$ is the primary solid particle diameter without isothermal holding, $\mathrm{D}_{\mathrm{t}}$ is the average particle size after isothermal holding for $\mathrm{t}$ seconds, and $\mathrm{K}$ is the coarsening rate constant). It illustrates that the regression coefficients are close to $1(0.9901)$ when the isothermal holding temperature is $600^{\circ} \mathrm{C}$. Hence, the optimal isothermal holding time of the A356 aluminum alloy semisolid slurry is $3 \mathrm{~min}$ when the isothermal holding temperature is $600^{\circ} \mathrm{C}$.

During the process of SIM, the melt temperature of the A356 aluminum alloy decreases rapidly duo to heat absorbance after adding the self-inoculants into the melt, in consequence, a large number of high melting points and "large sized atomic clusters" will be produced in the local position of the melt, which will be used as the nucleation substrates. On the other hand, the self-inoculants can be regarded as the heterogeneous nucleation substrates to increase the nucleation rate after adding into the melt. The above process is called primary inoculation process. Then the melt flows through the fluid director. In this process, the solidified shell is formed rapidly on the director surface due to the chilling of the low temperature of director surface. Subsequently, free grains and dendritic fragments are formed and involved in the melt when the subsequent melt scour and shear the solidified shell strongly, and finally evolve into rose-shape and fine dendritic primary particles. During this process, the temperature of the melt decreased and the undercooling of the semisolid slurry increased due to the heat transfer and convection, leading to the dendritic primary particles survived, which is called the process of secondary inoculation. In the outlet of the director, turbulence occurs when two streams of the melt are converged, promoting thermal field and concentration field of the melt to be homogeneous. The microstructures of solidified shell in different positions of the director have been observed as shown in Fig. 7. The microstructure of solidified shell in the inlet of the director is large dendrites. When the melt passes through the director, the microstructure of solidified shell in the outlet is rose-like crystals. Finally, when the slurry collected in the accumulator, the microstructure exhibits the fine equiaxed crystals due to the fusing of rose-like crystals (as shown in Fig. 4(a)).

In the isothermal holding process, the dendrite roots are fused due to the solute enrichment, promoting the formation of irregular particles. As a result, the interfacial energy is increased due to the increasing amount of particles. Primary 


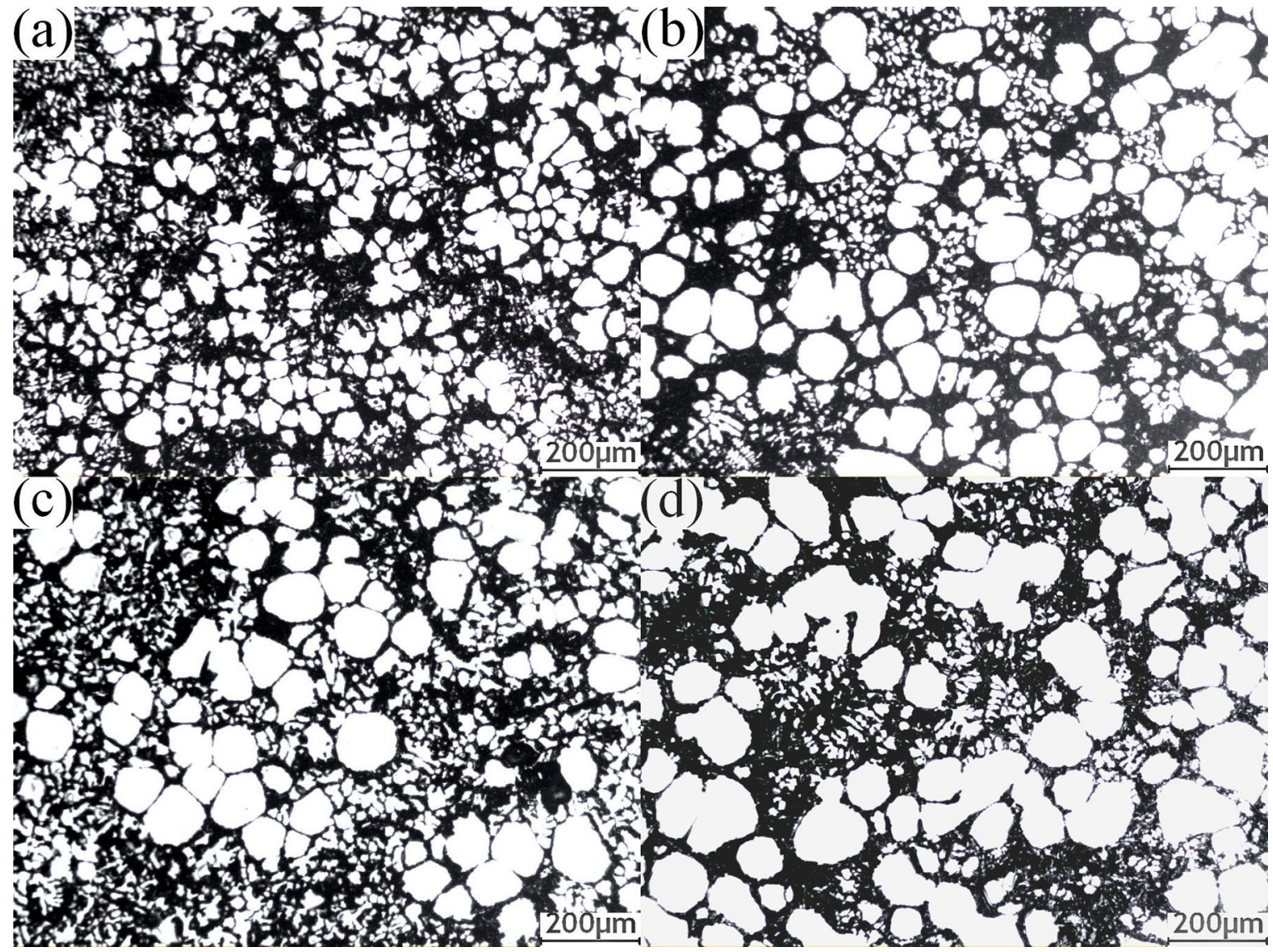

Figure 4. Water-quenched microstructures of A356 aluminum alloy semisolid slurry after isothermal holding at $600^{\circ} \mathrm{C}$ holding for (a) 0 $\min$, (b) $3 \mathrm{~min}$, (c) $5 \mathrm{~min}$ and (d) $10 \mathrm{~min}$

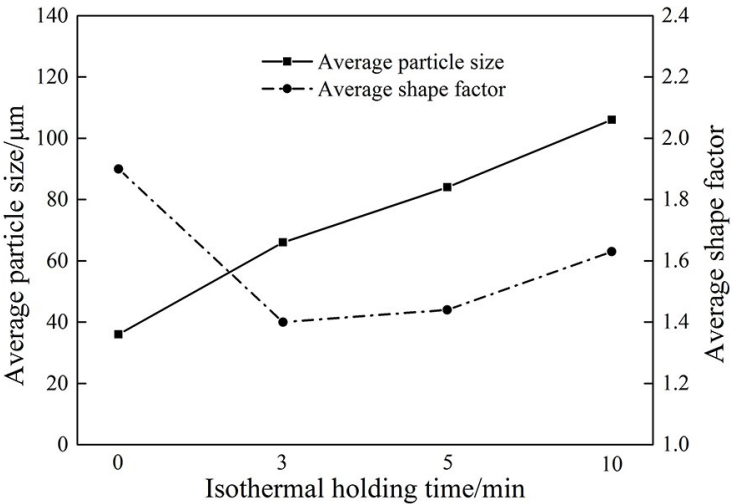

Figure 5. Effects of different isothermal holding time on average particle sizes and shape factors of primary particles

particles, as the substrates to absorb solute atoms from liquid phase, are rounded and spherical under the influence of the driving force (the interfacial energy can be reduced as far as possible). Consequently, primary particles are spheroidized and the sizes are increased with the extension of isothermal holding time. However, it can be seen different diameters of spherical primary particles after the slurry isothermally held for a short time, owing to the different sizes of initial dendrite fragments. The solute concentration of liquid phase

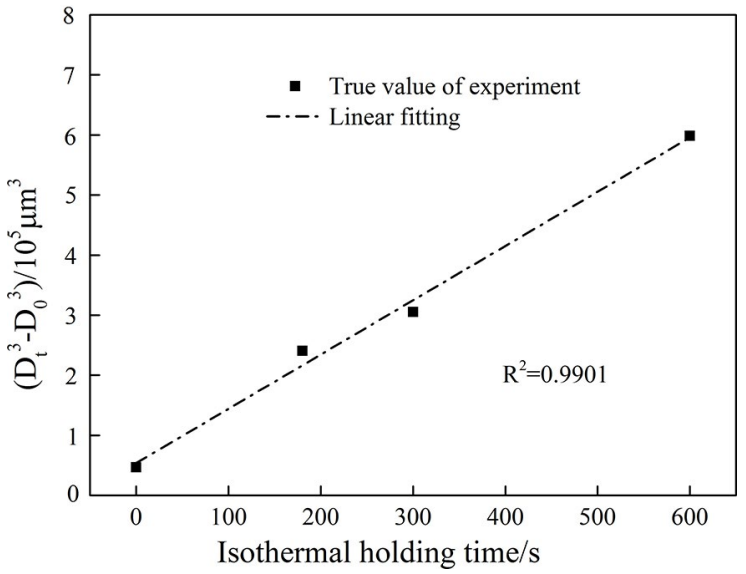

Figure 6. Linear regression of the growth of primary particles

around small particles is lower than large particles, with the further extension of the holding time, Si elements will continue to diffuse from large particles to small particles, while the $\mathrm{Al}$ elements have the opposite diffusion path. As a result, large particles become larger and small particles become smaller even melted and disappeared, which is called Oswald ripening ${ }^{22}$. The solid phase diffusion causes the coarsening of the primary solid particles in the slurry, and 
makes them rounded. After isothermal holding for a relative long time (such as $10 \mathrm{~min}$ ), the merging phenomena occur among primary particles, resulting in the generation of the " 8 " shaped particles and some irregular particles.

\subsection{Morphologies of primary particles in different forming processes}

After selecting the optimal parameters of the A356 aluminum alloy semisolid slurry, the microstructures of rheo-forming in different forming processes are shown in Fig. 8. It can be seen that the size of the primary particles in different forming processes are gradually increasing

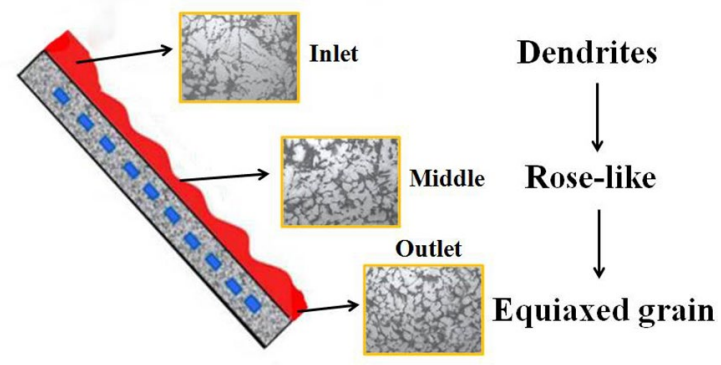

Figure 7. Microstructure evolution of the A356 aluminum alloy during the secondary inoculation process. with the decrease of cooling rate provided by the mould. Meanwhile, sharp corners around the primary particles are obviously occurring with the decrease of cooling rate, and the irregular degree of primary particles becomes more and more obvious with the decrease of cooling rate.

The results of particle sizes and shape factors of primary particles in different forming processes are measured as shown in Fig. 9. It can be seen that the average primary particle size of rheo-diecasting is about $54.58 \mu \mathrm{m}$, with the shape factor of about 1.34 . The average primary particle size of permanent mould castings are $66.73 \mu \mathrm{m}$ and $96.58 \mu \mathrm{m}$, and the corresponding shape factors are 1.88 and 2.1 , respectively. While in sand mould casting, the primary particle size and shape factor are both largest with the value of $127.68 \mu \mathrm{m}$ and 2.46, respectively. As is shown in Fig. 8 and Fig. 9, both the primary particle sizes and shape factors are gradually increasing with the increase of cooling rate, indicating that the cooling rate has obvious effect on morphology of primary particles.

In this experiment, the different forming processes for semisolid rheo-forming can be regarded as the effect of cooling rate on rheo-forming. When the semisolid slurry is poured into the mould, the $\mathrm{Al}$ element of remaining liquid will attach to primary particles, leading to the growth of primary

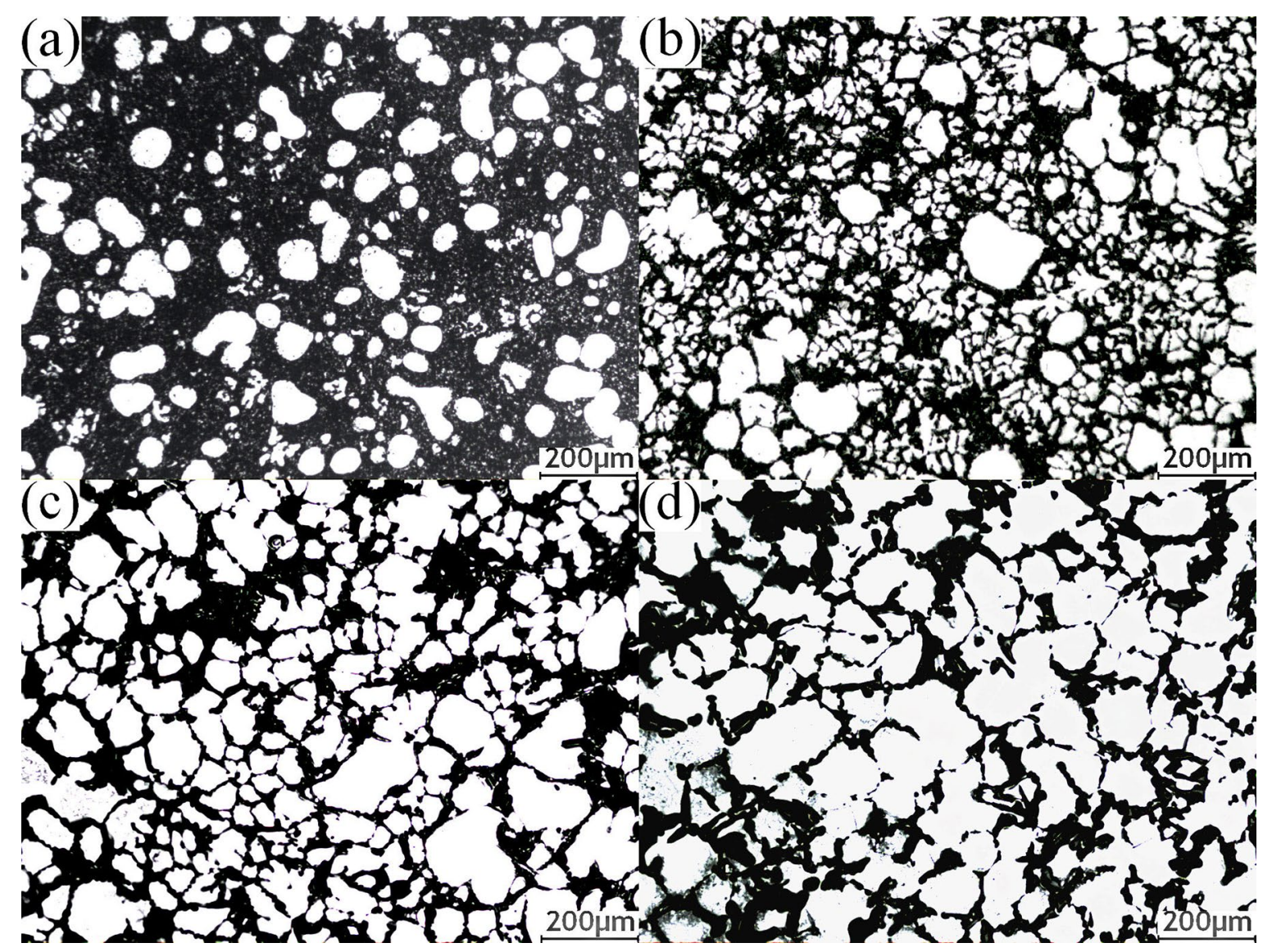

Figure 8. Microstructures in different forming processes (a) rheo-diecasting; (b) permanent mould casting with diameter of 15mm; (c) permanent mould casting with diameter of $50 \mathrm{~mm}$ and (d) sand mould casting with diameter of $50 \mathrm{~mm}$. 


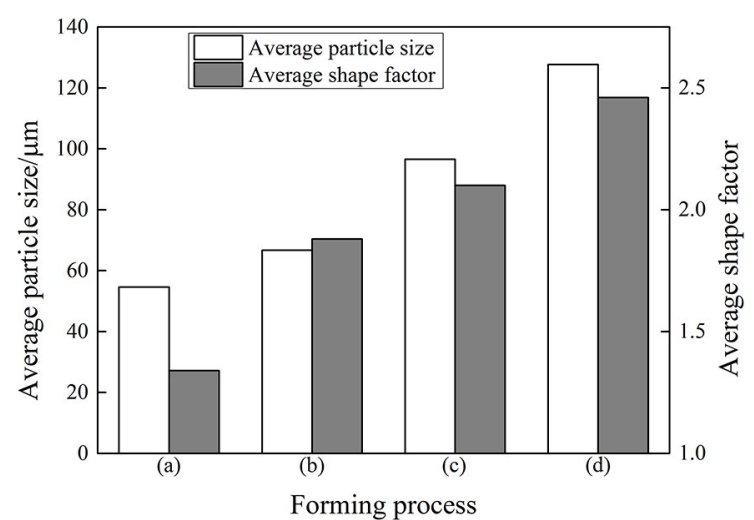

Figure 9. Values of primary particle sizes and shape factors in different forming processes (a) rheo-diecasting with the thickness of $2 \mathrm{~mm}$; (b) permanent mould casting with diameter of $15 \mathrm{~mm}$; (c) permanent mould casting with diameter of $50 \mathrm{~mm}$; (d) sand mould casting with diameter of $50 \mathrm{~mm}$.

particles. During the rheo-diecasting process, the cooling rate provided by cavity is too fast to form attachment growth obviously, and the primary particles have no time to grow larger. For the permanent mould casting with the diameter of $15 \mathrm{~mm}$, the growth of primary particles will be limited due to the relative large cooling rate $\left(17.2^{\circ} \mathrm{C} / \mathrm{s}\right)$. Moreover, the merging phenomenon between two touched particles will not be completely carried out during the solidification process of slurry. For the permanent mould casting with the diameter of $50 \mathrm{~mm}$, the primary particles will grow larger than the mould with the diameter of $15 \mathrm{~mm}$ due to its relative lower cooling rate. Meanwhile, the merging phenomenon of touched primary particles are clearly observed due to the decreased cooling rate $\left(5.2^{\circ} \mathrm{C} / \mathrm{s}\right)$. With the further reducing of cooling rate $\left(2.9^{\circ} \mathrm{C} / \mathrm{s}\right)$ provided by sand mould casting, the primary particles are growing largest, and the merging phenomenon of touched primary particles are obviously. Therefore, it can be proved that the merging phenomena of touched primary particles are increasing with the decrease of cooling rate. As a result, primary particle sizes and shape factors are gradually increasing with the increase of cooling rate.

On the other hand, the growth of primary particles depends on the attachment of $\mathrm{Al}$ atoms in remaining liquid of semisolid slurry. In the process of secondary solidification, due to attachment growth around the $\alpha_{1}$ particles, temperature fluctuations exist in the front of the interface, and then generate perturbation ahead of the interface of growing crystals. As the solidification process continue, the enriched layer of solute atoms will be generated stably in the liquid phase ahead of the interface, which will lead to a zone of "constitutional undercooling" ahead of crystals. At this point, the liquid phase in this zone is in a quasi steady state, meaning that there is a driving force which causes perturbation to grow.
Consequently, the temperature gradient and the concentration gradient in the liquid phase will increase at the tip of the solid liquid interface, which will also make the liquidus gradient increase. Then, the zone of "constitutional undercooling" will be saved, making the perturbation around $\alpha_{1}$ particles continuously grow. Due to the different cooling rate, the perturbation will be grown into different morphologies. For the permanent mould casting with the diameter of $15 \mathrm{~mm}$, the perturbation can be solidified to "cellulation". While in the other two mould, the perturbation can be solidified to "dentation" due to relative low cooling rate. Hence, sharp corners around the primary particles are obviously occurring with the decrease of cooling rate.

\subsection{Solidification behavior of secondary particles in different forming processes}

The rheo-forming by SIM includes two processes: primary solidification process and secondary solidification process. Secondary solidification starts when the semisolid slurry leaves the slurry collector. As shown in Fig. 8, the fine particles around primary particles are secondary particles, which are the results of solidification of remaining liquid. The clear SEM graphs of secondary particles in different forming processes are shown in Fig. 10, and the changes of morphologies and sizes of secondary particles can be seen obviously. It can be seen that the sizes of secondary particles are gradually increasing with the decrease of cooling rate, and the amount of secondary particles in the same areas are gradually decreasing with the decrease of cooling rate, indicating that the cooling rate has large effect on effective nucleation number.

Fig. 11 shows the measured results of particle sizes and shape factors of secondary particles in different forming processes. It can be seen that the average particle size of rheo-diecasting is $6.59 \mu \mathrm{m}$, with the shape factor of 1.49 . The average particle sizes of permanent mould casting are $27.91 \mu \mathrm{m}$ and $38.73 \mu \mathrm{m}$, and the corresponding shape factors are 1.91 and 1.66 , respectively. While in sand mould casting, the average particle size and shape factor are $53.25 \mu \mathrm{m}$ and 1.44 , respectively. It can be found from the results of Fig. 10 and Fig. 11 that the cooling rate of the mould also has obvious effect on sizes and morphologies of secondary particles.

Component analysis of primary particles, secondary particles and eutectic structures are carried out, in order to further explain the difference of the chemical composition in different forming processes, the results as shown in Table 2. It can be seen that the contents of $\mathrm{Si}$ in primary particles are lower than secondary particles. As the different forming processes, the contents of $\mathrm{Si}$ in both the primary particles and secondary particles are different. The contents of $\mathrm{Si}$ in primary particles are gradually increasing with the decrease of cooling rate. While in secondary particles, the contents of 

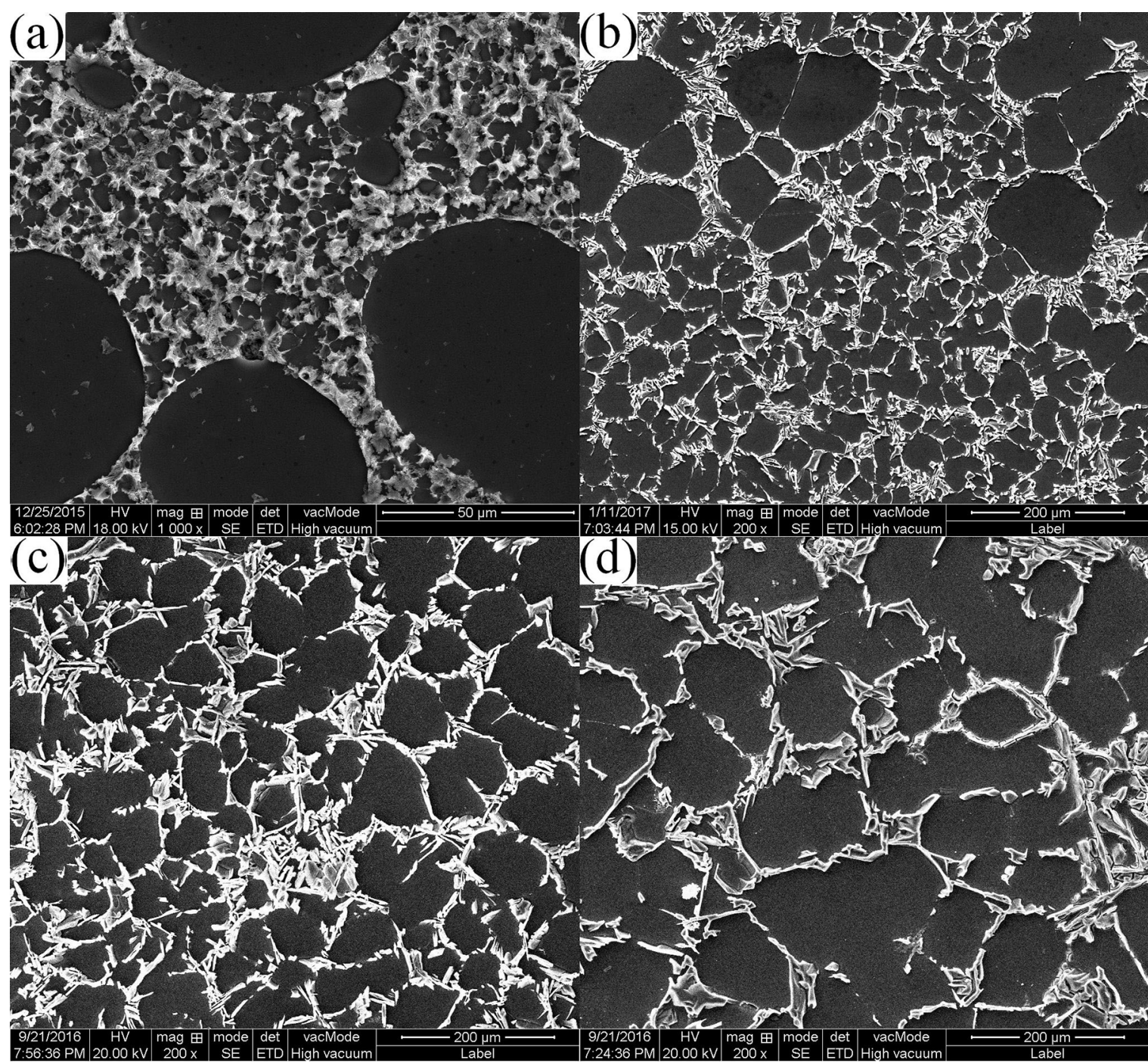

Figure 10. SEM graphs of rheo-forming in different forming process (a) rheo-diecasting with the thickness of $2 \mathrm{~mm}$; (b) permanent mould casting with diameter of $15 \mathrm{~mm}$; (c) permanent mould casting with diameter of $50 \mathrm{~mm}$; (d) sand mould casting with diameter of $50 \mathrm{~mm}$.

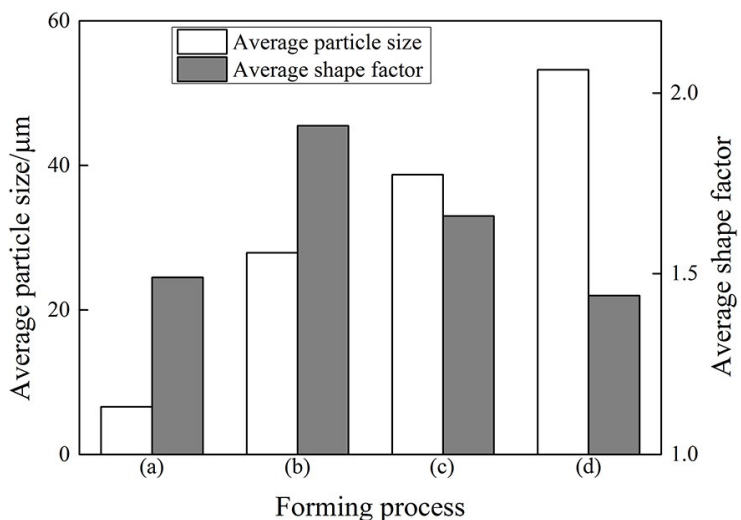

Figure 11. Values of primary particle sizes and shape factors in different forming processes (a) rheo-diecasting with the thickness of $2 \mathrm{~mm}$; (b) permanent mould casting with diameter of $15 \mathrm{~mm}$; (c) permanent mould casting with diameter of $50 \mathrm{~mm}$; (d) sand mould casting with diameter of $50 \mathrm{~mm}$.
Si are gradually decreasing with the decrease of cooling rate. Moreover, the content of $\mathrm{Mg}$ is 0.2 in secondary particles of process (d) while of others are 0 .

Like primary solidification process, the secondary solidification process also includes nucleation and growth. The nucleation rate expression is as follows ${ }^{23}$ :

$$
N=K \exp \left(\frac{-\Delta G}{k T}\right) \cdot \exp \left(\frac{-Q}{k T}\right)
$$

where $K$ is a constant, $\Delta G$ is the nucleation energy, $Q$ is the diffusion activation energy of atoms across the liquid/solid interface, $k$ is the Boltzmann constant, $T$ is thermodynamic temperature. For most of the alloy melt, the nucleation rate is increased gradually when the value of relative supercooling is below $0.15-0.25 T_{m}$ ( $T_{m}$ is the melting temperature of alloy). In this experiment, the supercoolings provided by 
Table 2. Average contents of $\mathrm{Al}, \mathrm{Si}$ and $\mathrm{Mg}$ element in different forming processes (a) rheo-diecasting with the thickness of $2 \mathrm{~mm}$; (b) permanent mould casting with diameter of $15 \mathrm{~mm}$; (c) permanent mould casting with diameter of 50mm; (d) sand mould casting with diameter of $50 \mathrm{~mm}$

\begin{tabular}{|c|c|c|c|c|c|c|c|c|c|}
\hline \multirow{2}{*}{$\begin{array}{l}\text { Different } \\
\text { forming } \\
\text { processes }\end{array}$} & \multicolumn{3}{|c|}{$\alpha 1$} & \multicolumn{3}{|c|}{$\alpha 2$} & \multicolumn{3}{|c|}{ Eutectic structure } \\
\hline & $\mathrm{Al}$ & $\mathrm{Si}$ & $\mathrm{Mg}$ & $\mathrm{Al}$ & $\mathrm{Si}$ & $\mathrm{Mg}$ & $\mathrm{Al}$ & $\mathrm{Si}$ & $\mathrm{Mg}$ \\
\hline (a) & 98.9 & 1.1 & 0 & 98.4 & 1.7 & 0 & 86.0 & 13.5 & 0.5 \\
\hline (b) & 98.9 & 1.1 & 0 & 98.4 & 1.6 & 0 & 78.2 & 21.4 & 0.5 \\
\hline (c) & 98.8 & 1.2 & 0 & 98.5 & 1.5 & 0 & 69.5 & 30.1 & 0.4 \\
\hline (d) & 98.6 & 1.4 & 0 & 98.3 & 1.5 & 0.2 & 37.0 & 62.8 & 0.2 \\
\hline
\end{tabular}

(a)

\section{8}

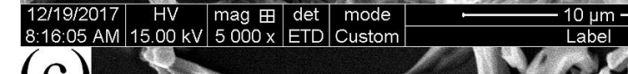

(c)
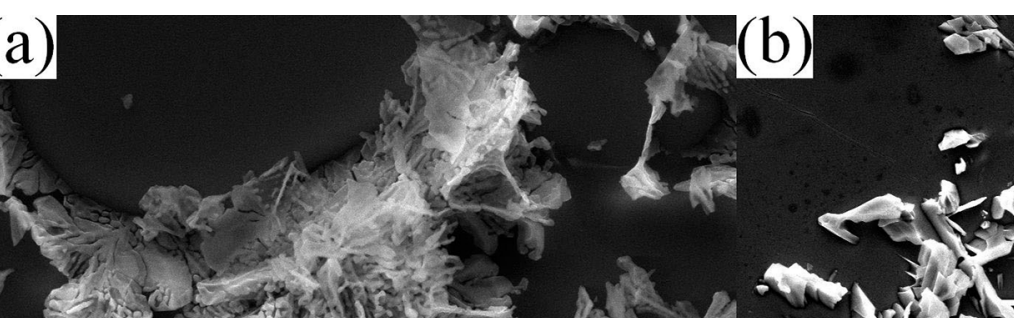

- is ats
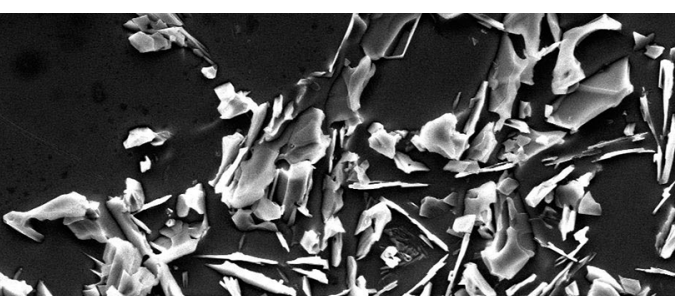

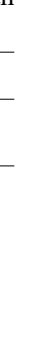


different mould are gradually increasing with the increase of cooling rate, leading to the different nucleation number.

The growth process starts when the nucleation process finishes. It can be simply understood that the secondary particles will be grown larger when the cooling rate is lower. The Mullins-Sekerka instability theory point out that a spherical crystal growing form a melt is morphologically unstable when its size exceeds a critical value $R c^{24}$ :

$$
\begin{aligned}
R c= & \frac{2 \Gamma\left(7+4 k_{s} / k_{l}\right)}{\left[\left(T_{m}-T_{\infty}\right) / T_{m}\right]}= \\
& \frac{2\left(\gamma_{s l} / L_{v}\right)\left(7+4 k_{s} / k_{l}\right)}{\Delta T / T_{m}}
\end{aligned}
$$

Where $T_{m}$ and $T_{1}$ are the melting point and melt temperature, $k s$ and $k l$ are thermal conductivity of liquid and solid $\mathrm{Al}$ at the melting point temperature, $\gamma_{s l}$ is the interfacial energy at the Solid/Liquid interface, and $L v$ the latent heat of fusion per unit volume of the solid. Substituting the thermal-physical values of pure aluminium ${ }^{12}$ into the above equation gives

$$
R c=\frac{5.12}{\Delta T}
$$

It can be seen that the critical value, $R c$, for stable spherical growth is sensitive to the undercooling, $\Delta T$. In the present study, it is difficult to directly measure the undercooling achieved by the $\alpha-\mathrm{Al}\left(\alpha_{2}\right)$ growing from the alloy melt, but the degree of undercooling is different due to the different cooling rate. The higher the cooling rate, the larger the degree of undercooling. Meaning that the high cooling rate (such as $17.2^{\circ} \mathrm{C} / \mathrm{s}$ ) leads to a small value of $R c$, on the contrary, the low cooling rate ( $\mathrm{such}$ as $2.9^{\circ} \mathrm{C} / \mathrm{s}$ ) leads to a large value of Rc. Namely, the secondary particles can grow into large spherical particles stably in a low cooling rate, and unstably grow into dendrites in a large cooling rate. Therefore, the measured data shows that the shape factors of secondary particles are gradually decreasing with the decrease of cooling rate, except for secondary particles in rheo-diecasting. During the rheo-diecasting process, the solidification process is finished before the unstable growth is completed, which is mainly due to the large cooling rate. Hence, the shape factor of secondary particles is not the largest.

Additionally, the solid fraction of aluminium alloy A356 at $600^{\circ} \mathrm{C}$ is measured to be $27 \%$ by Pandat (a thermodynamic calculation software). Assuming that the alloy contains only two kinds of elements, $\mathrm{Si}$ and Al (because the contents of other elements are little), the average solid solution of $\mathrm{Si}$ in the primary particles is $1.13 \%$, which is measured combining equilibrium phase diagram and Pandat. According to the conservation of mass ${ }^{25}$, the composition of the remaining liquid phase, $C_{l}$, can be expressed as:

$$
C_{l}=\frac{C_{0}-C_{s} f}{1-f}
$$

Where $C_{0}$ is the original content of the alloy, $f$ is solid fraction and $C_{s}$ is the composition of the solid phase. According to equation (4), the content of $\mathrm{Al}$ and $\mathrm{Si}$ in the remaining liquid phase are $91.7 \%$ and $8.3 \%$, respectively, indicating that the composition of the remaining liquid phase are deviated to the eutectic composition (12.6\%) compared to the original composition of alloy. The difference content of Si between primary particles and secondary particles in the experimental results indicate that primary particles are precipitated in the original composition of the A356 alloy, while secondary particles are formed in the remaining liquid. During the rheo-forming process, the large cooling rate $\left(1000^{\circ} \mathrm{C} / \mathrm{s}\right)$, making the $\mathrm{Si}$ atoms enriched ahead of the interface and have no time to diffuse, and eventually dissolve in secondary particles. While the $\mathrm{Si}$ atoms have enough time to diffuse in low cooling rate $\left(2.9^{\circ} \mathrm{C} / \mathrm{s}\right)$. Consequently, the contents of $\mathrm{Si}$ are gradually decreasing in primary particles with the decrease of the cooling rate, but increasing in secondary particles. Mg element is measured only in secondary particles of sand mould casting due to the fully diffusion of $\mathrm{Mg}$ in low cooling rate.

\subsection{Eutectic structures in different forming processes}

Fig. 12 shows the morphologies of eutectic $\mathrm{Si}$ in different forming processes. It can be seen that the cooling rates provided by different mould have obvious effect on morphologies of eutectic Si. When the cooling rate is about $1000^{\circ} \mathrm{C} / \mathrm{s}$, the eutectic $\mathrm{Si}$ is fine fibrous. As the cooling rate decreases from $17.2^{\circ} \mathrm{C} / \mathrm{s}$ to $2.9^{\circ} \mathrm{C} / \mathrm{s}$, the eutectic structures are incompact gradually, the morphologies of eutectic $\mathrm{Si}$ are mainly needle-like and small amount of block-like, and the sizes of the eutectic Si are gradually increasing with the decrease of cooling rate. The lamellar spacing of the eutectic Si in different forming processes is measured as shown in Fig. 13. It shows that the average lamellar spacing of the eutectic Si is gradually increasing with the decrease of cooling rate.

The formation of the intergranular eutectic Si phase between the $\alpha$-Al particles marks the completion of solidification in the rheo-forming process. $\mathrm{Si}$ is a faceted phase and $\mathrm{Al}-\mathrm{Si}$ eutectic is usually considered as an irregular eutectic. The morphology of eutectic silicon in as cast structure is coarse 


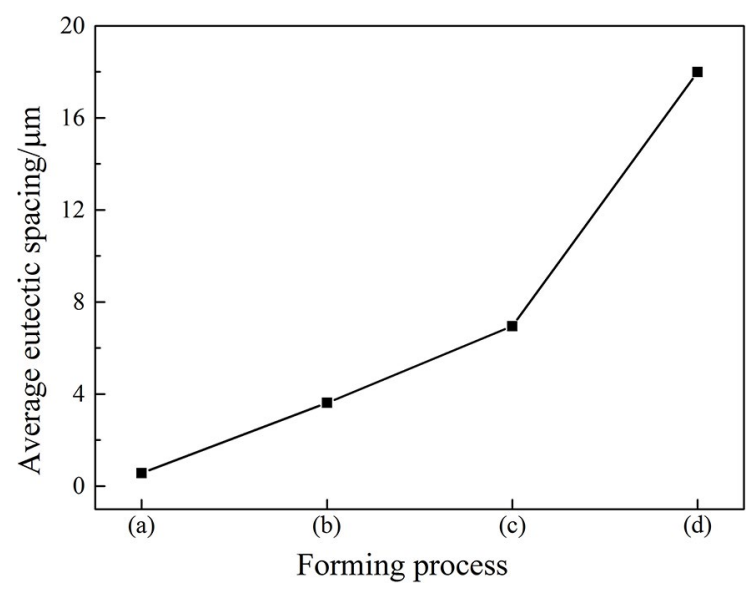

Figure 13. Average lamellar spacing in different forming processes (a) rheo-diecasting with the thickness of $2 \mathrm{~mm}$; (b) permanent mould casting with diameter of $15 \mathrm{~mm}$; (c) permanent mould casting with diameter of $50 \mathrm{~mm}$; (d) sand mould casting with diameter of $50 \mathrm{~mm}$.

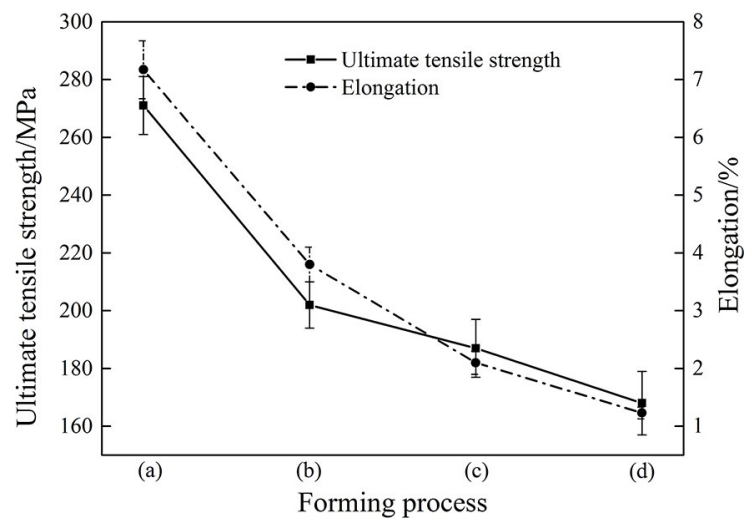

Figure 14. Mechanical properties of the components of different forming processes (a) rheo-diecasting with the thickness of $2 \mathrm{~mm}$; (b) permanent mould casting with diameter of $15 \mathrm{~mm}$; (c) permanent mould casting with diameter of $50 \mathrm{~mm}$; (d) sand mould casting with diameter of $50 \mathrm{~mm}$.

needle-like (as shown in Fig 11). According to the JacksonHunt theory of eutectic growth ${ }^{26}$, there is a relationship between eutectic spacing $\lambda$ and growth velocity $V$ :

$$
\lambda \sqrt{V}=\text { constant }
$$

with the constant being measured to be $25.2 \pm 3.2 \mu \mathrm{m}^{2 / 3} \mathrm{~s}^{-1 / 2}$ for Al-6Si-Al-12Si alloy ${ }^{27}$. The measured eutectic spacing is $0.57 \mu \mathrm{m}, 3.62 \mu \mathrm{m}, 6.95 \mu \mathrm{m}$ and $17.99 \mu \mathrm{m}$, respectively. According to Eq. (5), the eutectic spacing of the above values corresponds to a growth velocity of about $1489 \mu \mathrm{m} / \mathrm{s}$, $36.91 \mu \mathrm{m} / \mathrm{s}, 10.02 \mu \mathrm{m} / \mathrm{s}$ and $1.49 \mu \mathrm{m} / \mathrm{s}$ for different forming processes from (a) to (d), respectively, indicating that the cooling rate have great effect on growth velocity of eutectic silicon. Hence, the eutectic spacing are tightly as the cooling rate gradually increases.

\subsection{Mechanical properties of different forming processes}

As we all known, the microstructure has obvious effect on mechanical properties of metal materials. In present experiment, the ultimate tensile strength of specimens in different forming processes is tested as shown in Fig. 14. It can be obtained from Fig. 13 that both the ultimate tensile strength and the elongation are the highest when the component is fabricated by the process of rheo-diecasting, with the corresponding values are about $271 \mathrm{MPa}$ and $7.17 \%$, respectively. As the cooling rate provided by mould decreasing, the ultimate tensile strength decreases from $202 \mathrm{MPa}$ to $168 \mathrm{MPa}$ as well as the change tendency of the elongation (decreases from $3.80 \%$ to $1.23 \%$ ). It indicates that the forming press and cooling rate have obvious effect on the tensile strength and elongation of the semisolid forming component of A356 alloy. Namely, the finer the microstructures, the larger the tensile strength and elongation.

\section{Conclusions}

1. Semisolid slurry of A356 aluminum alloy can be prepared by Self-Inoculation Method at $600^{\circ} \mathrm{C}$. Primary $\alpha$-Al particles with fine and spherical morphology are uniformly distributed when the isothermal holding time of slurry is $3 \mathrm{~min}$.

2. The different forming processes for semisolid rheoforming can be regarded as the effect of cooling rate on rheo-forming, which has large effect on morphologies of primary particles. To be precise, primary particle sizes and shape factors are gradually increasing with the increase of cooling rate.

3. The different forming processes influences not only the nucleation process of the remaining liquid, but also the growth process of secondary particles. The sizes of secondary particles are gradually increasing with the decrease of cooling rate, and the amount of secondary particles in the same areas are gradually decreasing with the decrease of cooling rate.

4. The morphologies of eutectic Si are gradually coarsening from fibrous to needle-like in different forming processes, while the sizes and the lamellar spacing of the eutectic Si are gradually increasing with the decrease of cooling rate.

5. The mechanical properties of the semisolid forming components are gradually increasing with the increase of cooling rate and forming press. 


\section{Acknowledgments}

'The authors would like to acknowledge the project is supported by National Natural Science Foundation of China (No. 51464031) and the Youth Foundation of Hexi University (No. QN2018011).

\section{References}

1. Flemings MC. Behavior of metal alloys in the semisolid state. Metallurgical Transactions A. 1991;22(5):957-981.

2. Luo SJ, Jiang YZ, Li YF, Shan WW. Recognition of Semi-Solid Metal Forming Technologies. Special Casting \& Nonferrous Alloys. 2012;32(7):603-607.

3. Fan Z, Fang X, Ji S. Microstructure and mechanical properties of rheo-diecast (RDC) aluminium alloys. Materials Science and Engineering: A. 2005;412(1-2):298-306.

4. Mahathaninwong N, Plookphol T, Wannasin J, Wisutmethangoon S. T6 heat treatment of rheocasting $7075 \mathrm{Al}$ alloy. Materials Science and Engineering: A. 2012;532:91-99.

5. Zhu WZ, Mao WM, Tu Q. Preparation of semi-solid 7075 aluminum alloy slurry by serpentine pouring channel. Transaction of Nonferrous Metals Society of China. 2014;24(4):954-960.

6. Guan RG, Cao FR, Zhao ZY, Huang H, Zhang QS, Wang C. Effects of wavelike sloping plate rheocasting and spheroidisation on microstructures and properties of $\mathrm{Al}-18 \% \mathrm{Si}-5 \% \mathrm{Fe}$ alloy. The Chinese Journal of Nonferrous Metals. 2011;21(9):20842090.

7. Yuck JA, Martinez RA, Flemings MC. Development of the semi-solid rheocasting (SSR) process. In: Proceedings of the 7th International Conference on Semi-solid Processing of Alloys and Composites; 2002 Sep 25-27; Tsukuba, Japan. p. 659-664.

8. Pan QY, Findon M, Apelian D. The continuous rheoconversion process (CRP): A novel SSM approach. In: Proceedings of 8th International Conference on Semi-solid Process of Alloys and Composites; 2004 Sep 21-23; Limassol, Cyprus.

9. Kaufmann H, Mundl A, Potzinger R, Uggowirzer PJ, Ishibashi $\mathrm{N}$. An update on the new rheocasting development work for Al-and Mg-alloys. Die Casting Engineer. 2002;46(4):16-19.

10. Midson SP. Rheocasting processes for semi-solid casting of aluminum alloy. Die Casting Engineer. 2006;50(1):48-51.

11. Li YD, Yang J, Ma Y, Qu JF, Zhang P. Effect of pouring temperature on AM60 Mg alloy semi-solid slurry prepared by self-inoculation method (I). The Chinese Journal of Nonferrous Metals. 2010;20(6):1046-1052.

12. Hitchcock M, Wang Y, Fan Z. Secondary solidification behaviour of the Al-Si-Mg alloy prepared by the rheo-diecasting process. Acta Materialia. 2007;55(5):1589-1598.
13. Li YD, Chen TJ, MaY, Yan FY, Hao Y. Microstructural characteristic and secondary solidification behavior of AZ91D alloy prepared by thixoforming. The Chinese Journal of Nonferrous Metals. 2008;18(1):18-23.

14. Chen ZW, Zhang HF, Lei YM. Secondary Solidification Behaviour of AA8006 Alloy Prepared by Suction Casting. Journal of Material Science \& Technology. 2011;27(9):769-775.

15. Guan RG, Chen LQ, Li JP, Wang FX. Dynamical solidification behaviors and metal flow during continuous semisolid extrusion process of AZ31 alloy. Journal of Materials Science and Technology. 2009;25(3):395-400.

16. Zhao ZY, Guan RG, Wang X, Liu CM. Microstructure formation mechanism during a novel semisolid rheo-rolling process of AZ91 magnesium alloy. Acta Metallurgica Sinica (English Letters). 2013;26(4):447-454.

17. Xing B, Li YD, Ma Y, Hao Y. Effects of novel self-inoculation method on microstructure of AM60 alloy. China Foundry. 2011;8(1):121126.

18. Xing B, Li YD, Ma Y, Chen TJ, Hao Y. Evolution of rheocast microstructure of AZ31 alloy in semisolid state. China Foundry. 2013;10(4):221-226.

19. Li YL, Li YD, Li C, Wu HH. Microstructure characteristics and solidification behavior of wrought aluminum alloy 2024 rheo-diecast with self-inoculation method. China Foundry. 2012;9(4):328-336.

20. Li YD, Zhang XL, Ma Y. Effect of mixing rate and temperature on primary Si phase of hypereutectic $\mathrm{Al}-20 \mathrm{Si}$ alloy during controlled diffusion solidification(CDS) process. China Foundry. 2015;12(2):173179.

21. Kumar SD,Acharya M, MandalA, Chakraborty M. Coarsening Kinetics of Semi-solid A356-5wt\%TiB2 in situ Composite. Transactions of the Indian Institute of Metals. 2015;68(6):1075-1080.

22. Hardy SC, Voorhees PW. Ostwald ripening in a system with a high volume fraction of coarsening phase. Metallurgical Transactions A. 1988;19(11):2713-2721.

23. Hu GX, Cai X, Rong YH. Fundamentals of Materials Science. Shanghai: Shanghai Jiao Tong University Press; 2015.

24. Ji S, Qian M, Fan Z. Semisolid processing characteristics of AM series $\mathrm{Mg}$ alloys by rheo-diecasting. Metallurgical and Materials Transactions A. 2006;37(3):779-787.

25. Yang W, Liu F, Wang HF, Lu BP, Yang GC. Non-equilibrium transformation kinetics and primary grain size distribution in the rapid solidification of Fe-B hypereutectic alloy. Journal of Alloys and Compounds. 2011;509(6):2903-2908.

26. Jackson KA, Hunt JD. Lamellar and rod eutectic growth. Transactions of the Metallurgical Society of American Institute of Mining, Metallurgical and Petroleum Engineers. 1966;236(8):1129-1142.

27. Grugel R, Kurz W. Growth of interdendritic eutectic in directionally solidified Al-Si alloys. Metallurgical and Materials Transactions A. 1987;18(6):1137-1142. 